\title{
A PRELIMARY APPROACH FOR THE AUTOMATED RECOGNITION OF MALIGNANT MELANOMA
}

\author{
EZZEDDINE ZAGROUBA ${ }^{1}$ AND WALID BARHOUMI ${ }^{2}$ \\ ${ }^{1}$ Département des sciences de l'informatique, Laboratory LIP2, Faculté des Sciences de Tunis (FST), Campus \\ Universitaire, 1060 Tunis, Tunisia, ${ }^{2}$ Groupe du Recherche en Images et Formes de Tunisie (GRIFT), Laboratory \\ CRISTAL, Ecole Nationale des Sciences de l'Informatique (ENSI), Campus Universitaire, 2080 La Manouba, \\ Tunisia \\ e-mail: ezzeddine.zagrouba@ @sm.rnu.tn,walid.barhoumi@laposte.net \\ (Accepted March 15, 2004)
}

\begin{abstract}
In this work, we are motivated by the desire to classify skin lesions as malignants or benigns from color photographic slides of the lesions. Thus, we use color images of skin lesions, image processing techniques and artificial neural network classifier to distinguish melanoma from benign pigmented lesions. As the first step of the data set analysis, a preprocessing sequence is implemented to remove noise and undesired structures from the color image. Second, an automated segmentation approach localizes suspicious lesion regions by region growing after a preliminary step based on fuzzy sets. Then, we rely on quantitative image analysis to measure a series of candidate attributes hoped to contain enough information to differentiate melanomas from benign lesions. At last, the selected features are supplied to an artificial neural network for classification of tumor lesion as malignant or benign. For a preliminary balanced training/testing set, our approach is able to obtain $79.1 \%$ of correct classification of malignant and benign lesions on real skin lesion images.
\end{abstract}

Keywords: fuzzy region growing, Karhunen-Loève transform, melanoma recognition, neural networks.

\section{INTRODUCTION}

The malignant melanoma is the most dangerous human skin disease. It is the deadliest form of all skin cancers and arises from cancerous growth in pigmented skin lesion. The incidence has increased dramatically during the last years. In the US, the incidence of melanoma is rising more rapidely than any other cancer. Data base available on the internet (e.g. Nidus Information Services (2001): http://www.ucdmc.ucdavis.edu/) report that since the early seventies, the melanoma has increased $126 \%$ in USA. In Europe, the malignant melanoma incidence is increasing by $5 \%$ every year and it is responsible of $91 \%$ of deathly skin cancer (Sboner et al., 2001).

If early recognized, the melanoma can be excised and the patient can recover completely. So one should intensify the awareness of all citizens. Indeed, the curability of skin melanoma is nearly $100 \%$ if recognized early enough, and treated surgically (Pehamberger et al., 1987). The early diagnosis of malignant melanoma is therefore a crucial issue for dermatologists. As a step towards improving early interpretation, a number of diagnostic checklists have been proposed, including UK "Seven Point checklist" (Healsmith et al., 1994), the American " $A B C D$ " list (Stolz et al., 1994), and "ABCDE" list (Fitzpatrick et $a l ., 1988)$. The lists specify visual features associated with malignant lesions symptoms. Unfortunately, it can be difficult to interpret visually these features and then to recognize malignant pigmented lesion. Even experienced dermatologists have difficulties for distinguishing melanoma from other pigmented lesions of the skin, such as typical and atypical lesions whose are benign. In fact, correct detection rates based on clinical visual investigation is commonly about $65 \%$ (Lee, 2001).

This problem has stimulated interest in adjunctive diagnostic modalities that might facilitate clinical recognition of melanoma, including the automated interpretation of dermatoscopic color images with computerized image analysis. Thus, there has been increasing interest in computer-aided systems $(C A D)$ for the clinical diagnosis of melanoma as a support for the dermatologists in different analysis steps, such as the lesion boundary detection, the quantification of diagnostic features, the classification in different types of lesions, the visualization, etc. Over the last few years, many works have developed allowing diagnostically useful systems based on image processing and recognition algorithms for atypical melanoma lesion. Readers interested in the current state of the art in computer aided image analysis in melanoma research, can refer to our precedent paper (Zagrouba and Barhoumi, 2003a).

Typically, the whole process for color image processing for melanoma detection includes four 
main steps: image formation and preprocessing, segmentation, features extraction and classification of the lesion. In fact, after the acquisition and preprocessing of color images of the skin, the next step of the whole image analysis process is the segmentation of the lesion from the surrounding skin. The lesion areas and boundaries being clearly identified, various attributes of the lesion characteristic of the malignity symptoms must be measured. Such characteristic features will then be the raw input to a recognition algorithm classifying the lesion as melanoma or not. For a classification system to be successful, all four sub-tasks must be performed with care. The four steps are addressed in the next sections.

\section{IMAGE FORMATION AND PREPROCESSING}

\section{DATA DESCRIPTION}

Dermatologists commonly use slides for lesion image storage and visual comparison. In this work, these slides were digitalized with a $35 \mathrm{~mm}$ film scanner Nikon LS-1000. The resulting images that we used are BMP-format and are coded on 24 bits storing the three color components $r$ (red), $g$ (green) and $b$ (blue). These $r g b$ images are $150 \times 150 \times 3$ pixels in size, stored in $67 \mathrm{KBytes}$, with a spatial resolution of $0.0264 \mathrm{~cm} \times 0.0264 \mathrm{~cm}$ per pixel. Each image has one or more lesions located near the center and the lesions are surrounded by normal skin of variable hue. Lesions can vary in size, shape, color and saturation. Fig. (1) shows the images of 4 different lesions. Lesions $a$ and $b$ are benign nevus, however, lesions $c$ and $d$ are malignant melanomas (Fig. 1). Other features, such as hairs and pigments, can be observed in the images which can confuse the further analysis of the images. Thus, a preprocessing step is needed in order to ameliorate the image quality. It consists in reducing noise, strongly present in dermatoscopic images, and to enhance edges in order to eventually facilitate the separation between the lesion and the surrounding skin.

In our system, we applied a median filtering for minimizing the influence of small structures (like thin hairs) and isolated islands of pixels (like small air bubbles) in the segmentation result. For images including thick hairs with a color hue similar to the one of the lesion and thus irremovable by the median filter (e.g. Fig. 1d), a specific hair removal technique (called DullRazor (Lee et al., 1997)) is applied. The last preprocessing step in our system is the application of the Karhunen-Loève transform that enhances the edges towards making easier the extraction of the lesion from the surrounding skin.

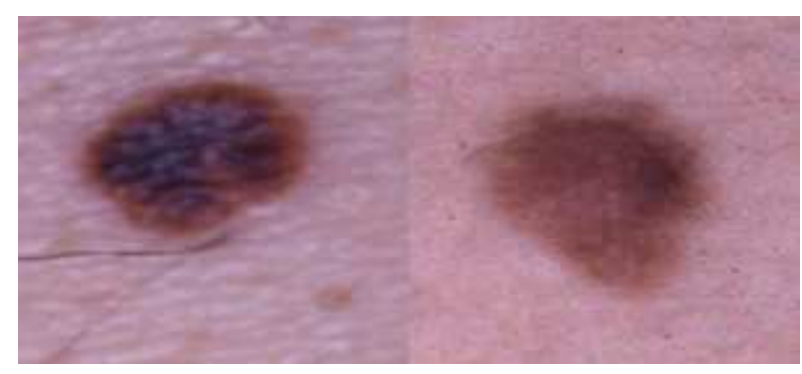

(a)

(b)

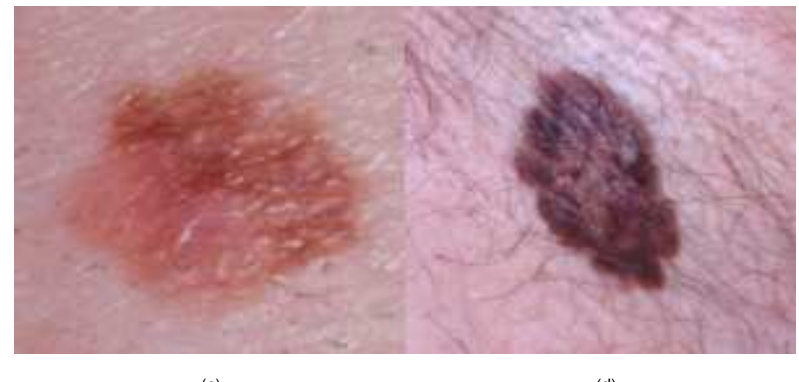

(c)

Fig. 1. Color images of lesions. (a-b): benign nevus, $(c-d)$ : melanomas.

\section{MEDIAN FILTERING}

Small structures and artifacts should be removed from skin images towards reducing the oversegmentation while at the same time preserving edges. These artifacts can be considered as impulsive noise and can be reduced using a median filter (HintzMadsen et al., 1996) (e.g. Fig. 2).

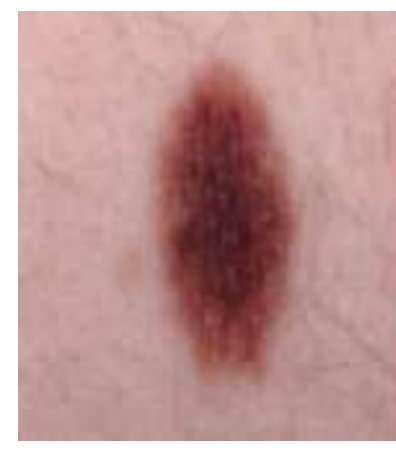

(a)

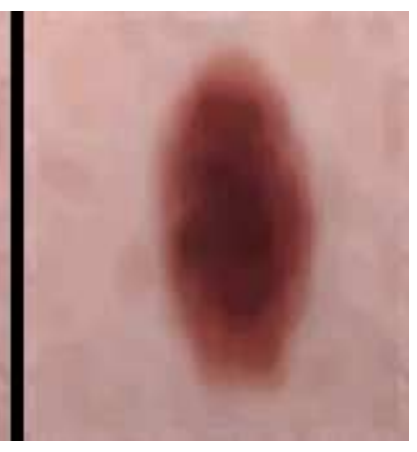

(b)
Fig. 2. Median filtering. (a) original image. (b) result after median filtering: inner structures and thin hairs have been smoothed out. 


\section{THICK HAIRS REMOVAL}

This process is necessary only for images covered by thick hairs where the median filtering is insufficient for hair suppression (e.g. Fig. 3). For these images, more than $50 \%$ of the pixels in the current $5 \times 5$ pixel neighborhood scanned by the median filter could be hair pixels. The median value would be then representative of the hairs. Thus, the median filtering can intensify the undesired pixels which may degrade the segmentation process.

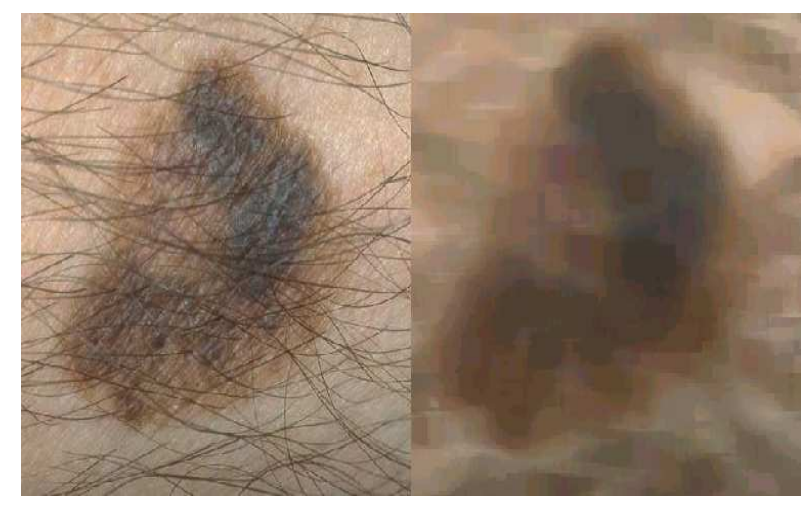

Fig. 3. Insufficiency of median filtering with a fixed $5 \times 5$ neighborhood for thick hair removal. (a) original image. (b) result after median filtering: some hair structures are intensified.

Of course, shaving the hairs before imaging sessions may be a solution. However, this solution not only adds extra costs and time to the image session, but also is uncomfortable and impractical especially for multiple lesions or total-body nevus imaging (Voight and ClaBen, 1995). Hence, in spite of its algorithmic complexity, the preprocessing technique called DullRazor (Lee et al., 1997) appeared to us as the best solution, from a practical point of view, for thick hair removal. It consists in identifying hair areas and replacing hair pixels with nearby non-hair pixels. In fact, for every pixel $p$ a generalized grayscale morphological closing operation is applied on the three color bands separately in order to localize the dark hairs. In practice, for every color band $b_{k}(k \in\{r, g, b\})$, this operation approximates thick hairs shapes by smoothing out low intensity values along fixed-size structure elements in horizontal, diagonals and vertical directions $\left(e_{0}, e_{45}, e_{135}\right.$ and $\left.e_{90}\right)$ representing classical hair forms. The generalized grayscale closing image $G_{k}$ is obtained by taking the maximum response from the individual closing operations for each color band (Eq. 1). Then, a binary hair mask $H_{k}$ image is created by thresholding $G_{k}$. This hair mask divides the hair and non-hair regions into disjoined areas. Finally, the global binary hair mask $(H)$ is the union $\oplus$ of all three hair masks $H=H_{r} \oplus H_{g} \oplus H_{b}$ (e.g. Fig. 4a).

$$
\begin{gathered}
\forall k \in r, g, b, \forall p, G_{k}(p)=\left|b_{k}(p)-\max \left(\mathbf{c}_{p}\right)\right|, \\
\mathbf{c}_{p}=\left\{\left[b_{k} \bullet e_{0}\right](p),\left[b_{k} \bullet e_{45}\right](p),\right. \\
\left.\left[b_{k} \bullet e_{90}\right](p),\left[b_{k} \bullet e_{135}\right](p)\right\},
\end{gathered}
$$

where $\bullet$ denotes the grayscale closing operation.

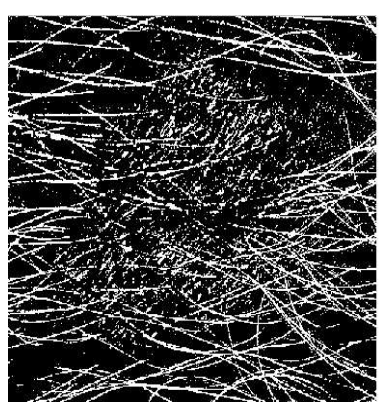

(a)

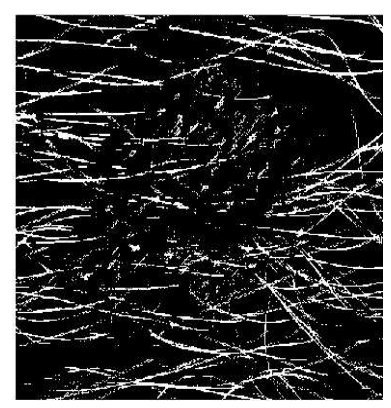

(b)
Fig. 4. Tick hairs removal. (a) hair mask $H$ of Fig. $3 a$. (b) the cleaned mask $H^{\prime}$ relatively to $H$. (c) cleaned image of Fig. $3 a$.

Before the replacement is performed, each pixel in the hair mask is checked to ensure that it is located within a thick and long structure (i.e hair structure); otherwise, the pixel is rejected as noise. In fact, for each pixel inside the hair region $H$, line segments are drawn in 8 directions, up, down, left, right and the four diagonals, radiating from the concerned pixel until the line segment reaches the non-hair region. These 8 line segments form 4 straight lines centred at the pixel. The length of each line is calculated and the longest one is noted. In our experiments, the longest line must be longer than 75 pixels and other lines must be shorter than 35 pixels (e.g. Fig. 4b). Then, a cleaned mask $H^{\prime}$ is obtained. In the last step, every pixel $\operatorname{Im}(i, j)$ verified to be inside a hair structure is replaced by the bilinear 
interpolation $\operatorname{In}(i, j)$ of the two nearby non-hair pixels $\operatorname{Im}\left(i_{1}, j_{1}\right)$ and $\operatorname{Im}\left(i_{2}, j_{2}\right)$ along the shortest line (Eq. 2).

$$
\begin{aligned}
\operatorname{In}(i, j)=\operatorname{Im}\left(i_{1}, j_{1}\right) * \frac{d_{2}\left((i, j),\left(i_{2}, j_{2}\right)\right)}{d_{2}\left((i, j),\left(i_{1}, j_{1}\right)\right)}+ \\
\quad+\operatorname{Im}\left(i_{2}, j_{2}\right) * \frac{d_{2}\left((i, j),\left(i_{1}, j_{1}\right)\right)}{d_{2}\left((i, j),\left(i_{2}, j_{2}\right)\right)},
\end{aligned}
$$

with $d_{2}$ is the euclidean distance defined by (Eq. 3):

$$
d_{2}\left(\left(i_{1}, j_{1}\right),\left(i_{2}, j_{2}\right)\right)=\sqrt{\left|i_{2}-i_{1}\right|^{2}+\left|j_{2}-j_{1}\right|^{2}} .
$$

\section{KARHUNEN-LOĖVE TRANSFORM}

The next preprocessing step consists in applying the Karhunen-Loève transform aiming to facilitate the segmentation process by enhancing the edges in the image. The Karhunen-Loève transform, also called principal components analysis (Loève, 1998), is the projection of the three color components on the eignvectors of their covariance matrix. The covaraince matrix Cov of the three channels (RGB) is computed as expressed by Eq. (4):

$$
\operatorname{Cov}=\frac{V \cdot V^{t}-m_{V} \cdot m_{V}^{t}}{x \cdot y}=B \Lambda B^{t},
$$

where $(x, y)$ is the size of each color channel, $V$ is a $3 \times x y$ matrix of pixel-realizations of the 3 color channels (Eq. 5), $m_{V}$ is the vector mean of the color channels (Eq. 6), $B=\left[\begin{array}{lll}b 1 & b 2 & b 3\end{array}\right]$ is a matrix whose columns are the eigenvectors of the covariance matrix and $\Lambda$ a diagonal matrix containing the eigenvalues of Cov in decreasing order: $\lambda_{1} \geq \lambda_{2} \geq \lambda_{3}$.

$$
\begin{gathered}
V=\left(\begin{array}{cccccc}
r(1,1) & . . & r(1, y) & r(2,1) & . . & r(x, y) \\
g(1,1) & . . & g(1, y) & g(2,1) & . . & g(x, y) \\
b(1,1) & . . & b(1, y) & b(2,1) & . . & b(x, y)
\end{array}\right), \\
m_{V}=\frac{1}{x y} \cdot \sum_{i=1}^{x} \sum_{j=1}^{y}\left(\begin{array}{l}
r(i, j) \\
g(i, j) \\
b(i, j)
\end{array}\right) .
\end{gathered}
$$

The Karhunen-Loève transformation is given by (Eq. 7):

$$
\begin{aligned}
& \forall k \in\{1,2, \cdots, x y\}, z_{k}=B^{t}\left(v_{k}-m_{V}\right) \\
& \text { and } T=\left(\begin{array}{llll}
z_{1} & z_{2} \cdots & z_{x y}
\end{array}\right)=\left(\begin{array}{c}
t_{1} \\
t_{2} \\
t_{3}
\end{array}\right),
\end{aligned}
$$

where $v_{k}$ is the $k^{\text {th }}$ column vector in $V$ and $T$ contains what is known as the principal components. The $k^{t h}$ $(k=1,3)$ row of $T$, noted $t_{k}$, is referred to as the $k^{t h}$ principal component.
Due to the decreasing ordering of the eigenvalues and corresponding eignvectors, the first principal component $t_{1}$ will contain the maximum variance. Since most variation occurs at edges between lesion and surrounding skin, the first principal component is a natural choice for segmentation. In fact, most of the texture and structure information will be mapped onto the first principal component (e.g. Fig. 5). However, although others principal components contain a small portion of the total variation, they may express features hidden in the original images.

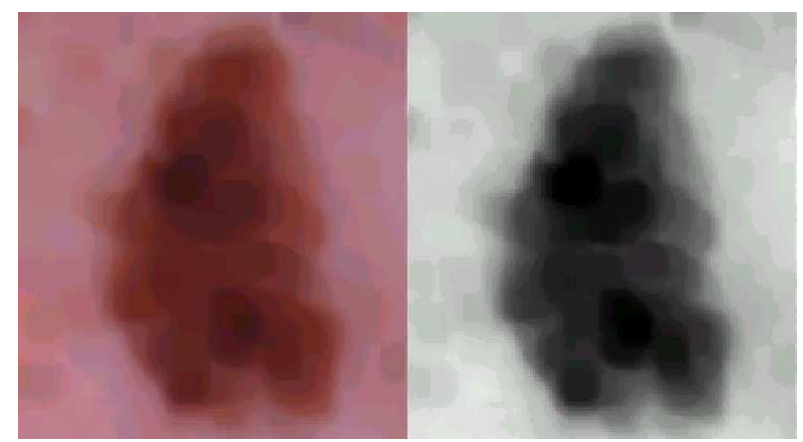

(a)

(b)

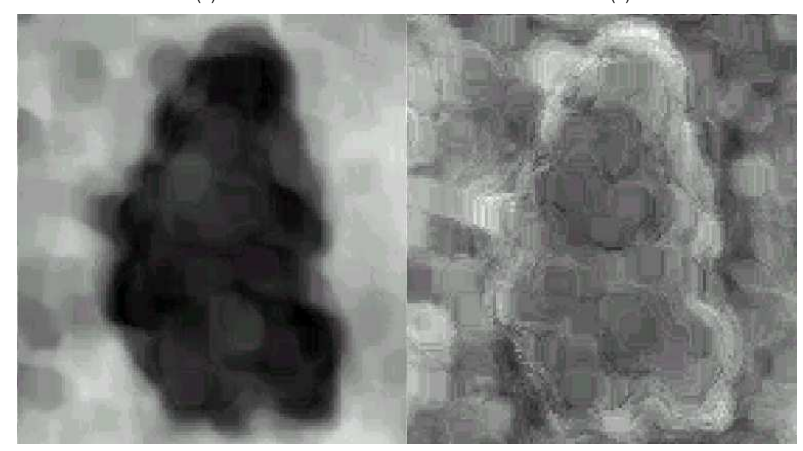

(c)

(d)

Fig. 5. Results of the Karhunen-Loève transform: (a) the original median filtered color image, (b) the first principal component accounting for $92.29 \%$ of the total variance, $(c)$ the second principal component$7.63 \%$, (d) the third principal lesion $-0.08 \%$.

\section{SEGMENTATION PROCESS}

Several studies in the literature address the segmentation of dermatoscopic images, with most of them relying on color and grayscale thresholding (Khanfir et al., 2002). However, the majority of these techniques are unable to define a criterion to separate with precision the pigmented lesion from the background healthy skin. It is due to the low contrast and the fuzzy nature of the boundaries of malignant melanomas. We propose an automated segmentation 
approach to localize suspicious lesion region in dermatoscopic images. It consists in determining the boundary of the lesion by region growing after an initial step based on fuzzy sets to enhance the lesion region of interest (ROI).

\section{PREPROCESSING BASED ON FUZZY SETS}

A typical first principal component of a median filtered dermatoscopic image consists of a very light background and a dark skin lesion with even darker areas inside. Herein, and as done elsewhere (Zagrouba and Barhoumi, 2002; Rangayyan et al., 1997), we have decided to characterize the lesion ROI working directly and only on the gray level intensities rather than using other measurements (e.g. local gradients). Then, a representative gray level $g l$ of the lesion must be chosen.

In agreement with the intuitive idea that the relevant classes may correspond to the dominant peaks in the gray level histogram, our objective is to obtain a non ambiguous bimodal histogram expressing the two following classes: lesion (class 1 ) and surrounding skin (class 2). Along that line, starting from the noisy bimodal histogram $h$ of the $t_{1}$ image (Fig. 6a), we applied a succession of local smoothings of type Eq. (8), yielding to the smoothed bimodal curve (Fig. 6b). This curve is characterized by two significant peaks $h_{1}$ and $h_{2}$ and their corresponding gray levels $g l_{1}$ and $g l_{2}$ assumed to be representative of the gray levels of the two classes. Since the lesion is always darker than the surrounding skin, its gray level $g l$ shall be the lower, i.e. the minimum of $g l_{1}$ and $g l_{2}$.

$$
h(i) \leftarrow \frac{1}{3}[h(i-1)+h(i)+h(i+1)] .
$$

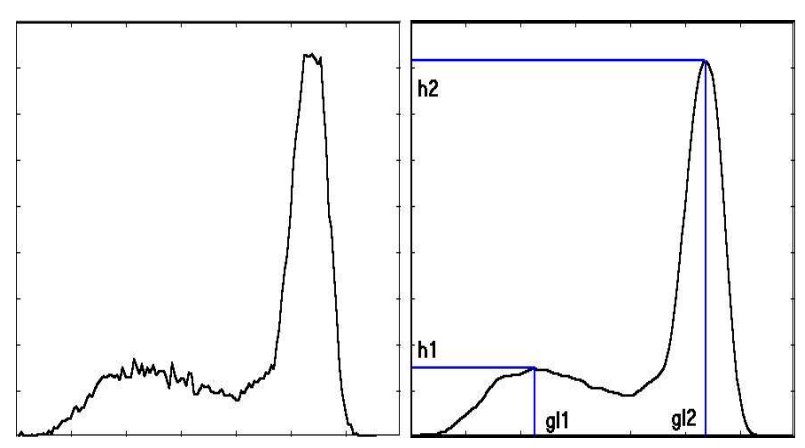

(a)

(b)

Fig. 6. histogram smoothing. (a) histogram of the first principal component image, (b) the smoothed bimodal curve.
The enhancement of the ROI may be achieved by defining an appropriate membership function that evaluates the similarity between the properties of any current pixel and those of the ROI itself $(g l)$. Thus, the original image $t_{1}$ will be mapped to a fuzzy set according to a symmetric membership function, decreasing monotonically from 1 to 0 , and assigning a membership of 1 to pixels of gray level $\mathrm{gl}$. The selected function has been defined after a study of many classical membership functions (Zagrouba and Barhoumi, 2003b) and is expressed by Eq. (9) where $\beta$ defines the opening of the membership function. The contrast of the ROI in the resulting image depends strongly upon the $\beta$ value. The larger $\beta$, the more the function is strict; the smaller $\beta$, the more the function is permissive. Fig. (7) expresses the resulting fuzzy set obtained with a small value for $\beta(\beta=0.007)$. The obtained fuzzy set represents pixels whose properties are close to the lesion with a high membership degree.

$$
m_{S}(p)=\frac{1}{2}\left[\frac{2-\beta^{2}|g l(p)-g l|^{2}}{1+\beta|g l(p)-g l|}\right] \text {. }
$$

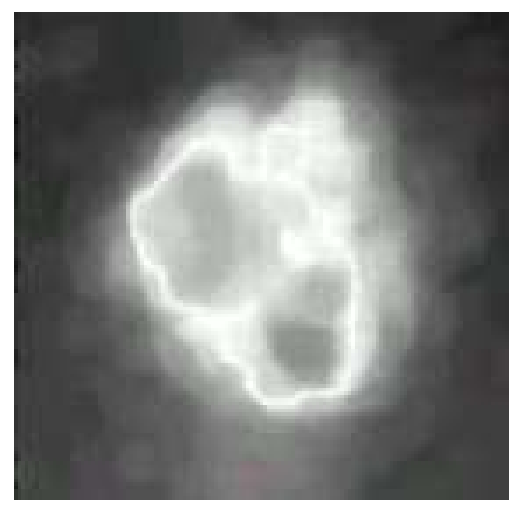

Fig. 7. Enhancement of the ROI: white pixels are close to 1 . Note that the fuzzy image has been rescaled to enhance the visual quality.

\section{LESION DETECTION BY REGION GROWING}

We propose to obtain the lesion ROI and its associated boundary by performing region growing upon the obtained fuzzy set image. Region growing is a segmentation technique that gathers pixels into an homogeneous region according to a similarity criterion. This algorithm needs a seed pixel that lies inside the ROI and a threshold $\theta$ as a stopping condition. It starts with the seed pixel which represents the first approximation of the ROI. Four-connected neighboring pixels that are above the threshold are labeled as one, the neighbors of these pixels 
are inspected and the procedure continues. If the connected pixel is less than the threshold, it is labeled as zero, indicating a boundary pixel, and its neighborhood are not processed. The recursive process continues until all connected pixels fail the test of inclusion in the region.

In order to optimize the region growing results, we wish to select the center of a homogeneous area as the seed pixel. For this, given the set $\xi$ of pixels having $g l$ as gray level, the seed pixel $S$ is selected according to the criterion defined by Eq. (10) (Fig. 8).

$$
|g l-m(S)|=\min _{p \in \xi}|g l-m(p)| .
$$

Where $m(p)$ is the mean gray level of a $5 \times 5$ pixel neighborhood $V(p)$ centered at pixel $p$.

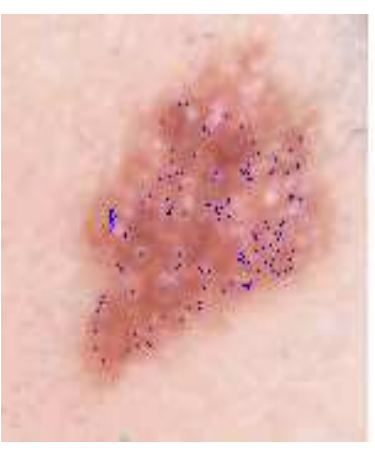

(a)

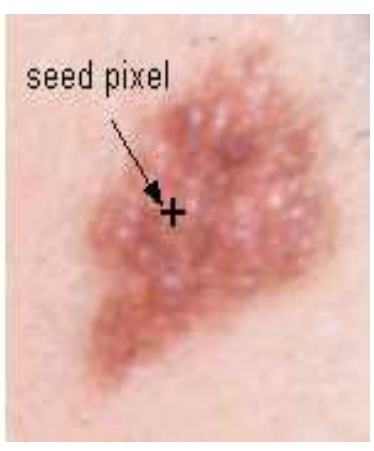

(b)

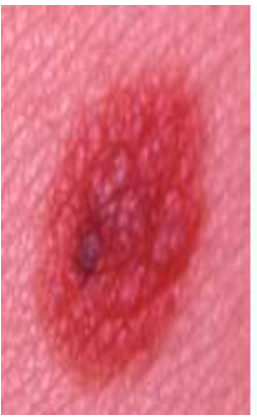

(a)

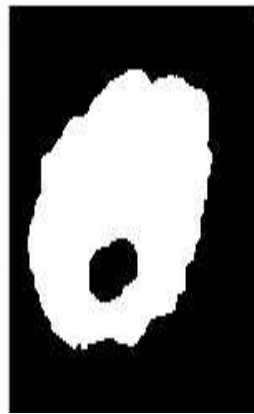

(b)

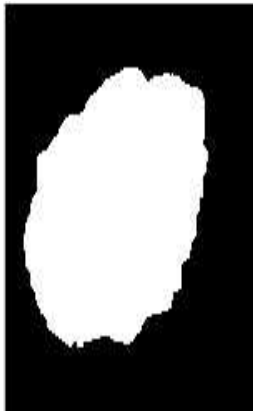

(c)
Fig. 9. Region growing process. (a) original image, (b) detection of the ROI lesion by region growing, (c) ROI after postprocessing of Dilatation-Erosion.

The parameters used in our segmentation algorithm, $\beta$ and $\theta$, must be adequately chosen in order to stop region growing at the boundaries of well-circumscribed lesions, where the membership values are expected to drop sharply across the lesion boundary (e.g. Figs. 10-11). In our study, optimal results are obtained with the values $\beta=0.007$ and $\theta=0.75$. In conclusion, our algorithm of segmentation is simple and easy to implement and will always produce connected region and closed boundary. The binary object and its closed boundary are the basis to compute the vector of numerical features, which is the purpose of the feature extraction module.

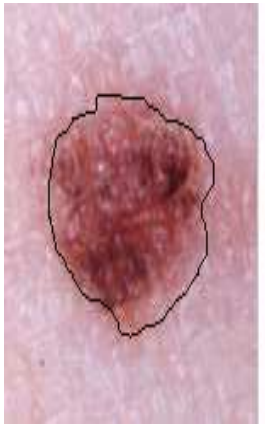

(a)

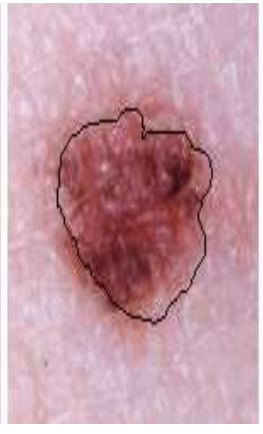

(b)

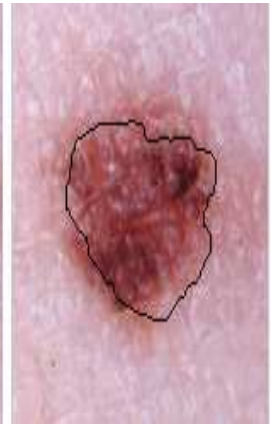

(c) surrounding skin) will be then treated by an algorithm of follow-up (Barhoumi and Zagrouba, 2002) applied to the border pixels of the lesion characterized by a local maximum of gradient. It allows the definition of a polygon representing an approximation of the lesion contour $C$.
Fig. 8. Seed pixel determination. (a) the set $\xi$ of lesion pixels having $g_{1}$ as gray level, (b) the seed pixel location.

After the region growing process, a postprocessing step of Dilatation-Erosion is done in order to remove isolated pixels inside the lesion's region (e.g. Fig. 9). At the end, the out-put of the lesion extraction procedure is the so-called binary plane (Fig. 9c) that separates lesion from the healthy surrounding skin. The resulting binary image (lesion $v s$. healthy 


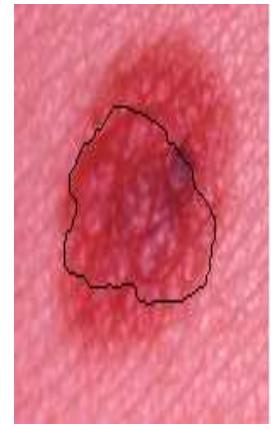

(a)

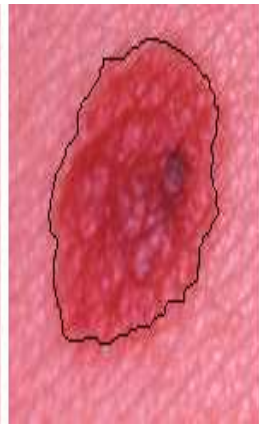

(b)

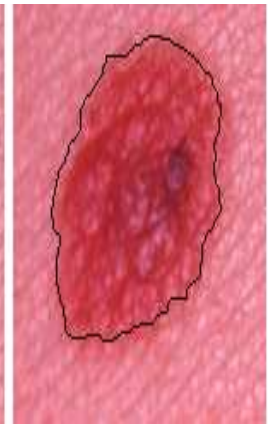

(c)
Fig. 11. Original rgb image superimposed with the boundary for various $\theta$ values $(\beta=0.007)$. (a) $\theta=$ 0.5 , (b) $\theta=0.7$, (c) $\theta=0.95$.

\section{DERMATOSCOPIC FEATURE DESCRIPTION}

The essential difficulty in melanoma recognition systems is to design robust and relevant parameters describing the lesions in order to ensure the separation between melanoma and benign lesions, in particular atypical benign ones which may be clinically mistaken for melanoma. We used the mnemonic device, $A B C D$, to describe several features that help to distinguish melanomas from noncancerous growths. The choice for the $A B C D$ rule is based primarily on the fact that the dermatologists we are working with use such rule. It shall be noticed that some studies report that the $A B C D$ rule may not yield the highest performance in melanoma diagnostic compared to alternative strategies such as stratification methods (Lorentzen et $a l .$, 2000). Yet, the diagnostic performance depends more on the selected attributes to represents the rule criteria, charaterizing the malignity symptoms, than on the used rule itself (Ganster et al., 2001) so that identifying the relevant $\mathrm{ABCD}$ features is definitely worth the effort. The different letters stand for the following criteria:

- Asymmetry (A): about half the time, a melanoma develops in an existing mole; in other cases, it arises as a new lesion that can resemble an ordinary mole. A noncancerous mole, however, is generally symmetric and circular in shape, while melanoma usually grows in an irregular, asymmetric manner.

- Border Irregularity (B): benign lesions generally have clearly defined borders. A melanoma, in contrast, often shows notched or indistinct borders that may signal ongoing growth and spreading of the cancer.
- Color Variation (C): one of the earliest signs of melanoma may be the appearance of various colors within the lesion. Because melanomas arise within pigment-forming cells, they are often varicolored lesions of tan, dark brown, or black, reflecting the production of melanin pigment at different depths within the skin.

- Diameter (D): early melanomas tend to grow larger than common moles and show typically at least a diameter of about $6 \mathrm{~mm}$.

$A B C D$ rules are commonly used by dermatologists. Yet a diagnosis made by a dermatologist based on the visual and qualitative evaluation of such criteria may be subjective (Schmid-Saugeon et al., 2003). Thus, our main purpose is to charaterize the $A B C D$ criteria by quantitative attributes measured by image analysis and then used as input to an automate classifier. In the literature, many attributes have been used to describe these features. A previous study by the authors (Zagrouba and Barhoumi, 2003a) of various attributes revealed some correlation between every single attribute and the melanoma diagnosis. However, each attribute alone is not sufficient to diagnose a lesion precisely. In other words, a combination of a set of $p(p>1$ in general) relevant attributes is necessary for the quantification of every feature and then for the diagnostic decision.

In Zagrouba and Barhoumi (2003b), we used 14 parameters describing the $A B C D$ rule and yielding $83 \%$ correct classification using a neural network classifier. This rate is related to a test subset of a series of images randomly selected from a database of 200 lesion images. However, this high number of attributes increases the classification complexity and the CPU time. We decided accordingly to define a reduced number of well-selected attributes permitting to obtain a higher correct classification rate while reducing the complexity and the CPU time by removing the redundant information. We will present, relatively to every feature, the adequate attributes that were chosen after many experiments and discussions with dermatologists. In fact, we realized a statistical study of 15 parameters (the 14 used in Zagrouba and Barhoumi (2003b) along with the diameter) representing the four criteria (Barhoumi et al., 2003). We classified a set of 100 lesion images by using a neural network classifier considering every attribute separately. For simplicity reasons, we chose a simple training set of images composed of 50 significant melanomas, 50 significant benign lesions and 0 atypical lesions images. Then, we measure for every attribute the correct classification rate $(T C R)$ relatively just to the chosen training set (this could explain the realtively high recorded $T C R$ values of 
some attributes, e.g. Fig. 12). We kept only the attributes having a correct classification rate greater or equal to $m_{T C R}$ with $m_{T C R}$ is the mean value of the $15 T C R$. In other words, we do not keep the attributes whose shall decrease the global diagnostic performance. Besides, we decided not to use the lesion diameter (attribute number 5) since it is indirectly integrated in the other attributes. Thus, the set of 9 attributes discussed below was selected.

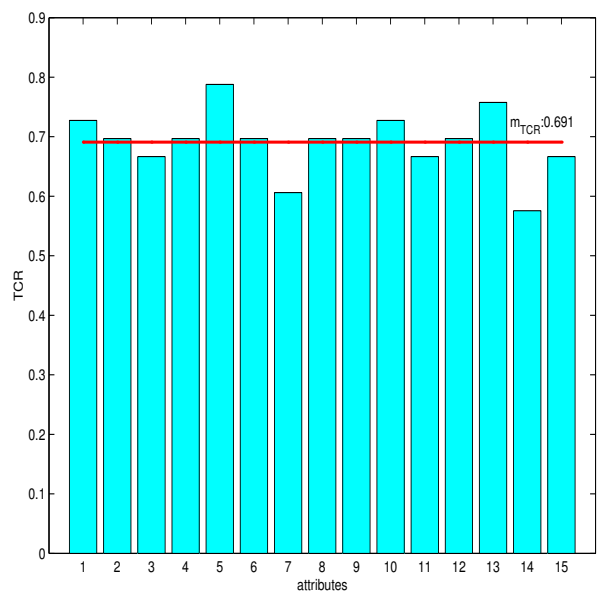

Fig. 12. Statical study of the correct classification rates of 15 attributes using a NN classifier.

\section{ASYMMETRY QUANTIFICATION}

In order to measure asymmetry, we rely hereafter on geometrical properties called inertial moments (Sonka et al., 1993). Such inertial moments are used to calculate two features: the asymmetry index $A I$ and the lengthening index $\AA$.

\section{Asymmetry index}

As far as asymmetry quantification is concerned, the origin of the local Cartesian basis is the center of mass $G$ of a current lesion $L$ described by a binary function $z(i, j)(z(i, j)=1$ if $(i, j) \in L, 0$ otherwise $)$. The quadratic inertial centred moment $I(\varphi)$ of the lesion $L$ with respect to an arbitrary axis through $G$ showing an angle $\varphi$ with the horizontal Cartesian axis $\Delta$ is given by Eq. (11):

$$
\begin{aligned}
I(\varphi) & =\sum_{(i, j) \in L} D_{\varphi}^{2}(i, j) \\
& =\sum_{(i, j) \in L}[-i \sin \varphi+j \cos \varphi]^{2},
\end{aligned}
$$

where $D_{\varphi}(i, j)$ is the distance between a current pixel $(i, j)$ and its projection on $\Delta$, along a direction normal to $\Delta$. The major principal axis is associated to the smallest inertia moment of $L$ and provides the longitudinal direction, $\varphi_{0}$, of $L$. It may then be obtained by computing the derivative of Eq. (11) and setting it to zero as expressed by Eq. (12):

$$
\frac{\partial I(\varphi)}{\partial \varphi}=0 \Longrightarrow \varphi_{0}=\frac{1}{2} \tan ^{-1}\left[\frac{2 m_{11}^{c}}{m_{20}^{c}-m_{02}^{c}}\right]
$$

where $m_{11}^{c}, m_{20}^{c}$ and $m_{02}^{c}$ represent the standard product moment, the quadratic moment with respect to the horizontal Cartesian axis $G_{x}$ and the quadratic moment with respect to the vertical Cartesian axis $G_{y}$.

The minor principal axis of $L$ associated to the direction $\varphi_{0}+\pi / 2$ yields the transverse direction of $L$ associated to the largest moment of inertia. The obtained longitudinal and transverse axes of $L$ may be used to calculate the asymmetry index. This is done by folding the lesion $L$ about these orthogonal axes and measuring the area of non overlap as expressed by Eq. (13) (e.g. Fig. 13):

$$
A I=\frac{1}{2} \sum_{k=1}^{2} \frac{\Delta A_{k}}{A_{L}}
$$

where the subscript $k$ identifies the principal axis (major vs. minor), $\Delta A_{k}$ is the corresponding nonoverlapping area of the folded lesion and $A_{L}\left(A_{L}=\right.$ $\left.m_{00}^{c}\right)$ is the lesion area.

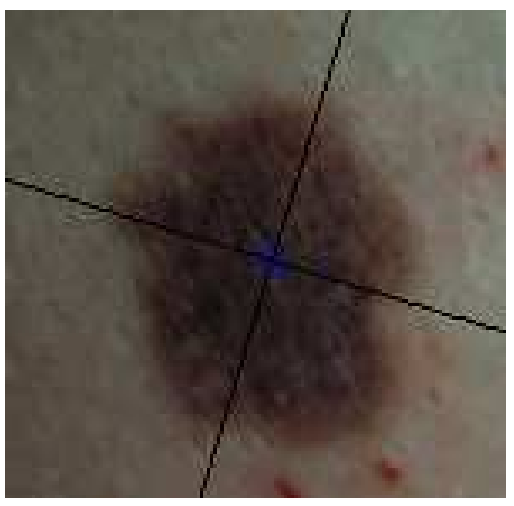

Fig. 13. Skin lesion image showing the two principal axes used to calculate asymmetry index. These axes define directions of smallest and largest moments of inertia. The asymmetry index for this lesion is 0.1796.

\section{Lengthening index}

This measure is used to describe the lengthening of a lesion, i.e. the degree of anisotropy of a lesion. Lengthening of a lesion is related to the eigenvalues $\lambda^{\prime}$, $\lambda^{\prime \prime}$ of the inertial tensor matrices. It is defined by the 
ratio of the inertial moment $\lambda^{\prime}$ about the major axis by the inertial moment $\lambda^{\prime \prime}$ about the minor axis (Eq. 14):

$$
\begin{aligned}
\AA & =\frac{\lambda^{\prime}}{\lambda^{\prime \prime}}, \\
\lambda^{\prime} & =\frac{m_{20}^{c}+m_{02}^{c}-\sqrt{\left(m_{20}^{c}-m_{02}^{c}\right)^{2}+4\left(m_{11}^{c}\right)^{2}}}{2}, \\
\lambda^{\prime \prime} & =\frac{m_{20}^{c}+m_{02}^{c}+\sqrt{\left(m_{20}^{c}-m_{02}^{c}\right)^{2}+4\left(m_{11}^{c}\right)^{2}}}{2} .
\end{aligned}
$$

\section{BORDER IRREGULARITY QUANTIFICATION}

The irregularity of a lesion border has been presented as a very significant diagnostic factor when assessing a lesion for malignancy (Keefe et al., 1990). In this work, we used five features to quantify the border irregularity. These features are: compactness index $C I$, fractal dimension $f d$, edge abruptness $\mathrm{Cr}$ and pigmentation transition $m_{e}$ and $v_{e}$.

\section{Compactness index}

The compactness index $(C I)$ (Eq. 15) is the most popular border shape measurement estimating the roundness of a $2 \mathrm{D}$ object. However, this measure is very sensitive to noise along the border which is amplified by the square term of the perimeter (Lee, 2001):

$$
C I=\frac{P_{L}^{2}}{4 \pi A_{L}},
$$

where $P_{L}$ is the perimeter of the lesion.

\section{Fractal dimension}

Extending from single-scale methods, the fractal dimension $(f d)$ is an elegant multi-scale method showing a high correlation with the human intuitive notion of roughness for curve lines (Tamura et al., 1978; Pentland, 1984). The fractal concept is based on the fact that different results are obtained when classical geometrical curves and fractal curves (such as coast lines) are measured using rulers of various size. For the first ones, the measured length $\ell$ converges to its true value as the ruler size $r$ decreases (Eq. 16). However, for the second ones $\ell$ increases as $r$ decreases. The inverse relationship between $\ell$ and $r$ is due to the fact that many bays and promontories smaller than the ruler size $r$ are unnoticed and omitted (Mandelbrot, 1982); they become noticeable if the unit size $r$ decreases:

$$
\ell=\lambda r^{1-f d},
$$

where $\lambda$ is a scaling constant and $f d$ is the characteristic of the coastline known as fractal dimension. The value of $f d$ is a fractional number that is larger than or equal to the Euclidean dimension of the object.

Many studies have used the fractal dimension to estimate the irregularity of lesion border ( $\mathrm{Ng}$ and Lee, 1996). The application of this measure to pigmented skin lesions showed that probability of a lesion being malignant increased with increasing fractal dimension, and hence border irregularity (Claridge et al., 1992; 1998). The box counting method is one of the most popular techniques to estimate the fractal dimension of a given curve $\ell$. The number of boxes $N(r)$ of size $r$ required to cover $\ell$ has the following relationship (Eq. 17):

$$
N(r) \propto \frac{1}{r^{f d}} .
$$

Thus, we used a square grid dividing the image into pixels of size $r \times r$ (e.g. Fig. 14). $N(r)$ was evaluated as the number of pixels containing a piece of the lesion boundary. Different pixel sizes $r$ were used and $f d$ was obtained as the slope of the regression line of $\log (r) v s$. $\log (N(r))$.

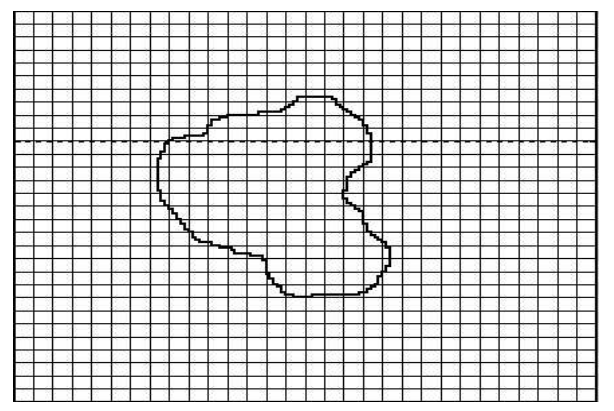

Fig. 14. Calculation of the fractal dimension $(f d)$ using the box counting method.

\section{Edge abruptness}

A lesion with irregular border has a large variance in the radial distance (i.e. the distance $d_{2}$ between its centroid $G_{L}$ and boundary $C$ ). Guthowicz-Krusin et al. (1997) estimated border irregularity by analyzing the variance of the radial distance distribution (Eq. 18):

$$
C r=\frac{\frac{1}{P_{L}} \sum_{p \in C}\left(d_{2}\left(p, G_{L}\right)-m_{d}\right)^{2}}{m_{d}^{2}},
$$

where $m_{d}$ is the mean of the distance $d_{2}$ between the boundary points and the centroid $G_{L}$. 


\section{Pigmentation transition}

This important feature describes the transition of the pigmentation between the skin lesion and the surrounding skin. A sharp abrupt edge suggests malignancy while a gradual fading of the pigmentation indicates a benign lesion (Hintz-Madsen et al., 1996). For this, we consider the luminance component $\operatorname{lum}(i, j)$ (Eq. 19) of the original color image as just an equally weighted sum of the three color components. Then, we estimate the gradient magnitude of the intensity component lum along the boundary $C$ of the skin lesion. We obtain a set of $K$ gradient magnitude values $e(k)(1 \leq k \leq K$, where $K$ is the number of boundary samples) describing locally the transition between the lesion and the skin background at each edge point. To describe more globally the transition, we used the mean $m_{e}$ and variance $v_{e}$ of the gradient magnitude values $e(k)$ describing globally the abruptness level and its variation (Eq. 20).

$$
\begin{gathered}
\operatorname{lum}(i, j)=\frac{1}{3}[r(i, j)+g(i, j)+b(i, j)] \\
m_{e}=\frac{1}{K} \sum_{k=1}^{K} e(k), \quad v_{e}=\frac{1}{K} \sum_{k=1}^{K} e^{2}(k)-m_{e}^{2} .
\end{gathered}
$$

\section{COLOR VARIATION QUANTIFICATION}

To further constrain the diagnosis, color variations in a lesion are here described by the color homogeneity $C_{h}$ and the correlation between geometry and photometry $C_{p g}$.

\section{Color homogeneity}

The luminance histogram of the lesion is divided into three intervals of equal length. The interval relating to the third smallest (resp. highest) luminance values defines the dark (resp. clear) areas in the lesion (Fig. 15); the intermediate interval relates to the rest of the lesion and does not participate in color quantification (Fig. 15). Then, color homogeneity $C_{h}$ (Taouil et al., 2002) is defined as the number of transitions clearer zone/darker zone and darker zone/clearer zone when scanning the lesion horizontally and vertically.

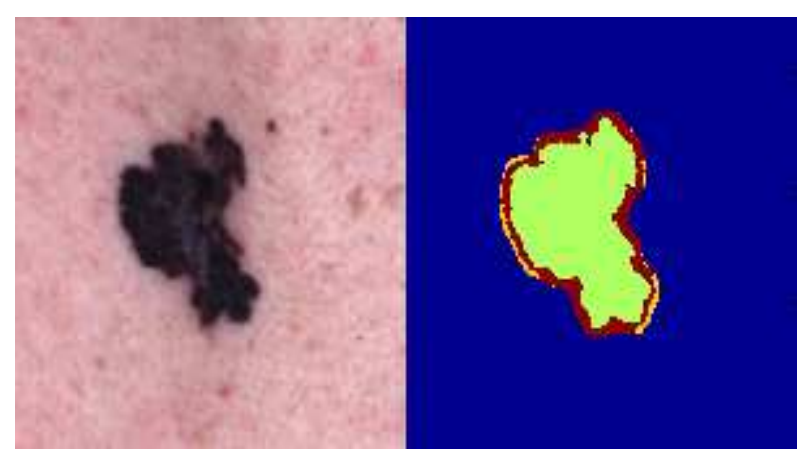

(a)

(b)

Fig. 15. Evaluation of the color homogeneity. (a) initial image, (b) the 3 color zones of the lesion used for the color homogeneity measure.

\section{Correlation between geometry and photometry}

This attribute evaluates the color distribution on the lesion. It consists in describing the evolution of the color level from the centroid $G_{L}$ towards the boundary of a lesion (Eq. 21). This value is greater for benign lesions since they have a targeted aspect, while low values are signs of malignancy.

$$
C_{p g}=\frac{1}{A_{L}} \cdot \sum_{p \in L} \frac{\left(\operatorname{lum}(p)-m_{l}\right) \cdot\left(d_{2}\left(p, G_{L}\right)-m_{d}\right)}{v_{l} \cdot v_{d}},
$$

where $m_{d}$ and $v_{d}$ are the mean and variance of the distance $d_{2}$ introduced in Eq. (18), $m_{l}$ and $m_{l}$ relating to the luminance.

\section{CLASSIFICATION PROCESS}

The last step of our strategy consists in training a classification process with the feature attributes selected above. On the one hand, several techniques of classification, such as K-Nearest Neighbors (KNN) (Ganster et al., 2001), Fuzzy C-Means (FCM) (Cucchiara and Grana, 2002) and decision tree (Sboner et al., 2001), have been applied to diagnose skin lesions. On the other hand, dermatologists base their clinical diagnostic decisions on experience as well as on complex inferences and extensive pathophysiological knowledge. Such experience can not be condensed into a small set of relations and this may limit the performance of algorithmic approaches (Ercal et al., 1994). Experience-based learning is the advantage of neural networks (Astion and Wilding, 1991) and this is a strong motive to using that type of approach in diagnostic applications such as 
ours. Besides, as illustrated by many previous studies (Binder et al., 1994; Ercal et al., 1994), neural networks (NN) may result in reduced error rates for the melanoma diagnosis problem when compared to strictly algorithmic approaches. NN approaches are also a very flexible means for mapping a fixed number of inputs into a set of discrete classes. Such characteristics motivated the use below of a NN approach towards mapping a series of sample attribute values into two diagnostic classes (cancerous lesions, healthy lesions).

\section{NEURAL NETWORK IMPLEMENTATION}

In the last two decades, NN have been extensively used for classification applications. In fact, the classical approach to classification is statistical and concerns the modeling of stationary class-conditional probability distribution by a set of basis functions (Ripley, 1996). The most common neural network architecture for supervised classification is the multilayer perceptron (Rumelhart et al., 1988). It possesses the important universal approximation capability, i.e., it may approximate any given function with arbitrary precision as long as the number of hidden-units is large enough. Thus, the multi-layer perceptron is the type of network that best fits our diagnosis application. After many tests with a different number of hidden layers, we decided to use a mono-layer perceptron yielding a satisfactory performance in a minimum training time. It may be represented by the network diagram in (Fig. 16).

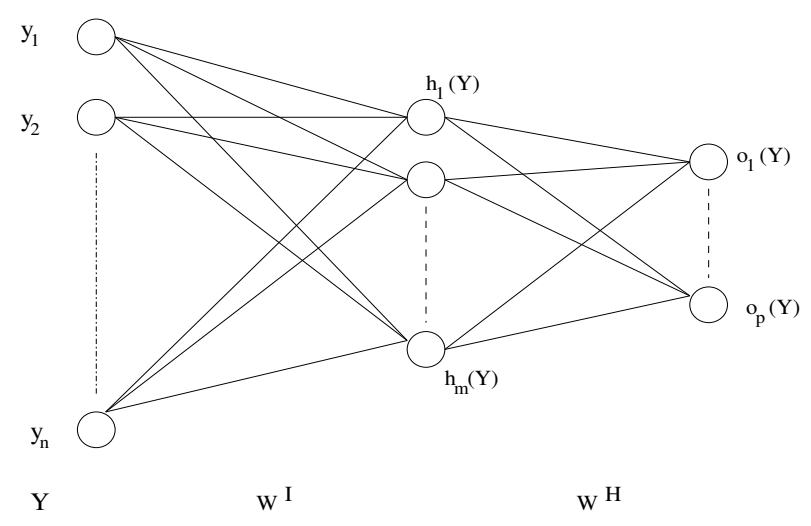

Fig. 16. Mono-layer perceptron structure.

The mono-layer perceptron is typically defined by sigmoidal hidden unit basis functions and by linear output functions. In this work, the chosen hidden activation function are hyperbolic tangent functions. Thus, the output of the hidden units for a pattern $Y$
$\left(Y=\left(y_{1}, y_{2}, \cdots, y_{n}\right) ; n\right.$ : number of input attributes) may be expressed as Eq. (22):

$$
\forall j \in\{1,2, \cdots, m\}, h_{j}(Y)=\tanh \left(\sum_{i=1}^{n} w_{j i}^{I} y_{i}+w_{j 0}^{I}\right),
$$

where $w_{j i}^{I}$ is the weight connecting input $i$ and hidden unit $j, w_{j 0}^{I}$ is the threshold for hidden unit $j$ and $m$ is the number of units in the hidden layer.

The hidden unit outputs are weighted and summed yielding the following unbounded network outputs Eq. (23):

$$
\forall k \in\{1,2, \cdots, p\}, \quad o_{k}(Y)=\sum_{j=1}^{m} w_{k j}^{H} h_{j}(Y)+w_{k 0}^{H},
$$

where $w_{k j}^{H}$ is the weight connecting hidden unit $j$ and the unbounded output unit $k, w_{k 0}^{H}$ is the threshold for the unbounded output unit $k$ and $p$ is the units number in the output layer.

In our case, the network architecture is defined by 9 input units representing the 9 selected attributes describing the malignity symptoms $(A I, \AA, C I, f d$, $C r, m_{e}, v_{e}, C_{h}$ and $C_{p g}$ ), $m$ hidden units and one linear output unit (thresholding function) producing a binary result ( 1 for a malignant lesion and 0 for a nonmalignant lesion). The attributes show different units and range values. Towards normalizing all attributes between 0 and 1 , an objective scaling was performed by calculating the corresponding $z$-scores (Kaufman and Rousseeuw, 1996).

After defining its architecture, the network is trained with supervision using the standard back-propagation algorithm. This algorithm allows the perceptron to approximate the classification function given the supervised training data. It is a process by which the free parameters of the perceptron (weights and thresholds) are adapted through the process of simulation using the training in supervised manner. Basically, back-propagation learning algorithm consists of two passes through the different layers of the network: a forward pass and a backward pass. In the forward pass, an input vector is applied to the network and its effect propagates through the network layer by layer. Then, a set of outputs is produced as the actual response of the perceptron. The weights of the network are all fixed during the forward pass. The backward pass starts at the output layer by passing error signals leftward through the perceptron and computing recursively the local gradient for each unit. Finally, this permit the perceptron weights to be all adjusted in accordance with an error-correction rule (Haykin, 1999). 
In our case, to approximate the classification function of a lesion as either benign or malignant, the back-propagation algorithm minimizes the squared error $\varepsilon_{r}$ between current outputs of the network and the desired ones given with the training data. The training process allows the perceptron to learn and gain experience about the melanoma diagnosis problem in order to automatically classify correctly skin lesions other than those included in the training set. The training continues till either the error $\varepsilon_{r}$ is lower than 0.1 or 10,000 iterations are executed which ensures the convergence of the perceptron to a global solution.

Furthermore, there are a number of arbitrary parameters whose values need to be defined carefully if the perceptron is to provide good performance. In particular, the number of hidden units largely affects the ability of the perceptron to determine the properties of patterns which are not members of the training set (Bostock et al., 1993). In fact, the gap between the approximation achieved by a neural network and the classification function to approach is inversely proportional to the number of hidden neurons (Barron, 1993). Unfortunately this result is not constructive, in the sense that it can only give vague estimations of the number of necessary hidden neurons. Thus, it does not exist at present time theoretical arguments to predict the number of necessary hidden units, to get a specified performance of the model, held account of the available data (Dreyfus, 2002). Thus, we varied the number of hidden units until optimal performance on the test set was obtained.

\section{EXPERIMENTS AND RESULTS}

The data set used herein consists of 200 images representing 75 representatives malignant lesions and 125 representatives benign ones. To evaluate the classifier performance of the designed perceptron, we used the holdout method (Huberty, 1994) which is the simplest kind of cross validation models. This method indicates how well the classifier will do when it is asked to make new predictions for data it has not already seen. It consists in separating the images data set into two disjoint and independent sets, called the training set $\mathbf{T} 1$ and the testing set T2. The classification function approximator fits a function using the training set only. Then, the function approximator is asked to predict the output values for the data in the testing set (it has never seen these output values before). The errors it makes are accumulated as before to give the mean absolute test set error, which is used to evaluate the classifier performance. The evaluation may depend heavily on which data images end up in the training set and which end up in the test set, and thus the performance evaluation of the perceptron may be significantly different depending on how the division is made. On the one hand, the size of the training set should be large enough to ensure a good classification rate. On the other hand, the size of the testing set should be also large enough to increase the confidence in the results (estimation of general performance). Herein we chose to use training and testing sets of comparable sizes; the following training T1 / testing T2 percentiles $(\kappa)$ were used: $40 / 60$, $50 / 50$ and $60 / 40$ (Table 1 ).

The obtained results are summarized in Table 1 for different number of hidden units $(n)$ so that the effect of architecture on performance could be assessed. This table records correct detection rates, using the perceptron classifier, on the training set $\mathbf{T} 1\left(T C R_{1}\right)$ and on the testing set $\mathbf{T} 2\left(T C R_{2}\right)$. Besides, accuracy of classification on the testing set is evaluated in terms of sensitivity $\mathrm{Sn}_{2}$ (percentage of malignant lesions correctly classified) and specificity $s p_{2}$ (percentage of benign lesions correctly classified). For each couple $(\kappa, n)$, the network weights are initialised randomly over $[-1,1]$ in every run and the final result, given in Table 1 , is computed as the average over a set of 10 runs. In Fig. 17, the results of a typical run of the designed algorithm of classification is shown.

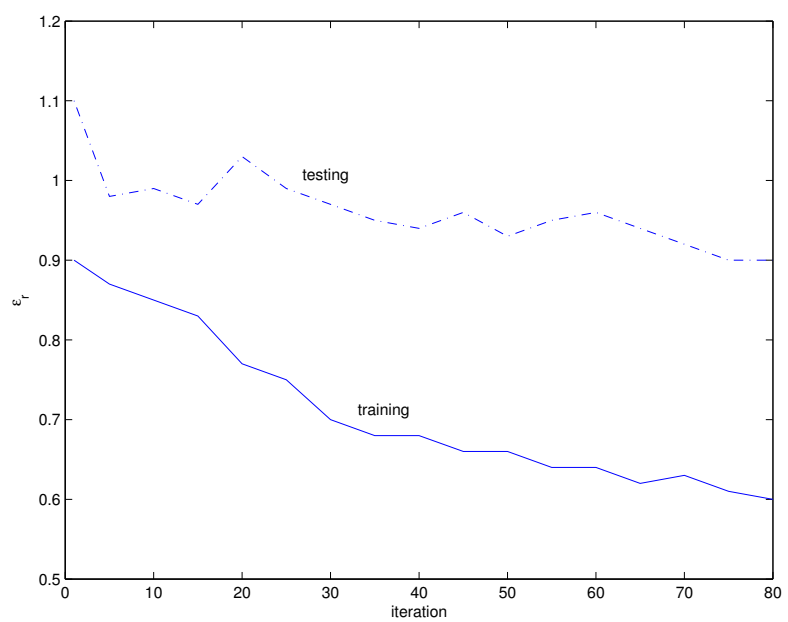

Fig. 17. Part of the results of a typical run of the melanoma recognition using the designed perceptron classifier: The development of the classification error during training of the perceptron by using backpropagation algorithm. Optimal weights are found by minimizing the validation error. Note that also the test errors decrease. 
Table 1. Neural network diagnostic results (percentage success rates) as a function of the number of hidden units $(n)$ and the training $\mathbf{T} 1 /$ testing $\mathbf{T} 2$ combination percentiles $(\kappa)$.

\begin{tabular}{|c|c|c|c|c|c|}
\hline$\kappa$ & $n$ & $T C R_{1}$ & $s n_{2}$ & $s p_{2}$ & $T C R_{2}$ \\
\hline $40 / 60$ & 3 & 69.9 & 56.0 & 73.2 & 64.6 \\
\hline $40 / 60$ & 4 & 85.7 & 68.3 & 77.1 & 72.7 \\
\hline $40 / 60$ & 5 & 81.3 & 66.5 & 80.3 & 73.4 \\
\hline $40 / 60$ & 6 & 81.0 & 76.6 & 79.2 & 77.9 \\
\hline $50 / 50$ & 3 & 74.7 & 63.6 & 70.9 & 67.25 \\
\hline $50 / 50$ & 4 & 88.6 & 69.5 & 75.1 & 72.3 \\
\hline $50 / 50$ & 5 & 74.2 & 67.0 & 76.4 & 71.7 \\
\hline $50 / 50$ & 6 & 81.7 & 61.3 & 76.8 & 69.05 \\
\hline $60 / 40$ & 3 & 71.8 & 61.2 & 77.4 & 69.3 \\
\hline $60 / 40$ & 4 & 94.5 & 75.1 & 83.1 & 79.1 \\
\hline $60 / 40$ & 5 & 86.1 & 67.4 & 76.5 & 71.95 \\
\hline $60 / 40$ & 6 & 93.7 & 74.5 & 81.2 & 77.85 \\
\hline
\end{tabular}

When we compare the success classification rates $\left(T C R_{1}\right.$ and $\left.T C R_{2}\right)$ for the three studied training/testing combinations $(\kappa)$, we can conclude that the best results are recorded for the $60 / 40$ combination. Independently of the hidden units number, this combination produces the higher mean of correct classification for both training and testing sets $(86.53 \%, 74.55 \%)$ when compared with the $50 / 50(79.8 \%, 70.08 \%)$ and $40 / 60(79.48 \%, 72.15 \%)$ combinations. This may be explained by the fact that the size of current database is so small, so that it appears more appropriate to exploit the maximum of data for the training step in order to generalize the network decision. Thus, a total of 120 (resp. 80) lesions were used in the training (resp. testing) set, including 45 (resp. 30) melanomas.

Given this training/testing combination, the perceptron architecture having 4 hidden units gave optimal performance, both on the training and testing sets $(72.14 \%, 67.05 \%)$ comparatively to these given with $3(89.6 \%, 74.7 \%), 5(80.51 \%, 72.35 \%)$ and 6 $(85.47 \%, 74.94 \%)$ hidden units architectures.

In conclusion, given the disposed database, the perceptron with one hidden layer composed of four units, trained on 120 lesion images and tested on 80 lesion images yielded the best results with correct classification rate $\left(T C R_{2}\right)$ of $79.1 \%$, sensitivity $\left(s n_{2}\right)$ of $75.1 \%$ and specificity $\left(s p_{2}\right)$ of $83.1 \%$. These results are comparable with the detection rates of very experienced dermatologists. However, the relatively higher rate of specificity when compared with the sensitivity rate is believed to be the consequence of the higher number of benign lesions than of melanoma images characterizing our data base.

\section{CONCLUSION AND FUTURE WORK}

In this study, we attempted to diagnose melanoma from color skin images using image processing techniques and an artificial neural network classifier. Towards that end, we relied on a preprocessing step essentially based on a median filter for its ability to remove noise and undesired components. Then, we performed a PCA like analysis to enhance edges. To extract the lesion from the image, an automated segmentation approach permitted to localize suspicious lesion region by region growing after a preprocessing step based on fuzzy sets. Then, after a study of several existing features describing the lesion malignity, a set of 9 attributes to distinguish melanoma from benign lesions was defined and methods to measure these attributes were described. Finally, a mono-layer perceptron was trained with these attributes in order to classify the lesion as melanoma or nonmelanoma.

Future studies will be carried out on the enlarged data set (483 dermatoscopic color images) so that a more rigorous evaluation of our approach can be made. Besides, we will try to extract a more reduced attributes set, using subset feature selection techniques, in order to keep the classification complexity low and to reduce the CPU time while at the same time preserving a high correct classification rate.

\section{ACKNOWLEDGMENTS}

We sincerely thank Mr. Patrick Vannoorenberghe, Laboratory PSI (Perception Systemes Information) - FRE CNRS2645, Univeristy of Rouen, France; who allowed us to recover the data set of color dermatoscopic images used in this article.

\section{REFERENCES}

Astion M.L, Wilding P (1991). Application of neural networks to the interpretation of laboratory data in cancer diagnosis. Clin Chem 38(2):34-8.

Barhoumi W, Zagrouba E (2002). Boundaries Detection Based on Polygonal Approximation by Genetic Algorithms. Knowledge-Based Intelligent Information Engineering Systems and Allied Technologies (KES 2002), Frontiers in artificial intelligence and applications, IOS Press 82(2):621-7.

Barhoumi W, Zagrouba E, Solaiman B, Ghorbel E (2003). Fusion de l'information par la théorie de l'évidence: application en diagnostic du mélanome. 
Proceedings of Conférence Internationale Sciences Electroniques, Techniques de l'Information et des Télécommunications, SETIT, Tunisia.

Barron A (1993). Universal approximation bounds for superposition of a sigmoidal function. IEEE Trans Inform Theory 39:930-45.

Binder M, Steiner A, Schwarz M, Knollmayer S, Wolff K, Pehamberger H (1994). Application of an artificial neural network in epiluminescence microscopy pattern analysis of pigmented skin lesions: A pilot study. Brit J Dermatol 130(4):460-5.

Bostock RT, Claridge E, Harget AJ, Hall PN (1993). Towards a neural network based system for skin cancer diagnosis, Third International Conference on Artificial Neural Networks, 372:215-9.

Claridge E, Hall PN, Keefe M, Allen J (1992). Shape analysis for classification of malignant melanoma. J Biomed Eng 14:229-34.

Claridge E, Morris JD, Hall PN (1998). Evaluation of border irregularity in pigmented skin lesions against a consensus of expert clinicians. Proceedings of Medical Image Understanding and Analysis, UK, 85-8.

Cucchiara R, Grana C(2002). Using the topological tree for skin lesion structure description. KnowledgeBased Intelligent Information Engineering Systems and Allied Technologies (KES 2002), Frontiers in artificial intelligence and applications, IOS Press, 82(1):166-70.

Dreyfus G (2002). Les réseaux de neurones : pourqoui et pour quoi faire. Réseaux de neurones - Méthodologies et Applications, Eds Eyrolles, 1-64.

Ercal F, Chawla A, Lee HC (1994). Neural network diagnosis of malignant melanoma from color images. IEEE Trans Biomed Eng 41(9):837-45.

Fitzpatrick TB, Rhodes AJ, Sober A, Mihm M (1988). Primary malignant melanoma of the skin: the call for action to identify persons at risk, to discover precursor lesions, to detect early melanomas. Pigm Cell Res 9(1):110-7.

Ganster H, Pinz A, Rohrer E, Wildling E, Binder M, Kittler H (2001). Automated melanoma recognition. IEEE Trans Med Imaging 20(3):233-9.

Guthowicz-Krusin D, Elbaum M, Szwaykowski P, Kopf AW (1997). Can early malignant melanoma be differentiated from atypical melanocytic nevus by in vivo techniques? Part II. Automatic machine vision classification. Skin Res Technol 3:15-22.

Haykin S (1999). Neural networks: A comprehensive fundation. Upper Saddle Rever, New Jersey: Prentice Hall.
Healsmith MF, Bourke JE, Graham-Brown RC (1994). An evaluation of the revised seven-point checklist for the early diagnosis of cutaneous malignant melanoma. Brit J Dermat 130:48-50.

Hintz-Madsen M, Hansen LK, Larsen J, Drzewiecki KT (1996). Detection of malignant melanoma using neural classifiers. in solving engineering problems with neural networks, International Conference on Engineering Applications using Neural Networks EANN, Turku, Finland, 395-8.

Huberty CJ (1994). Applied discriminant analysis. New York: John Wiley and Sons, Inc.

Kaufman L, Rousseeuw P.J (1990). Finding groups in data. New York: John Wiley and Sons, Inc.

Khanfir I, Kallel A, Taouil K, Bouhlel MS, Kammoun L (2002). Segmentation d'images par seuillage d'histogrammes : Application à l'analyses de mélanomes. JTEA'2002, Tunisia, 190-8.

Keefe M, Dick D, Wakkel R (1990). A study of the value of the seven-point checklist in distinguishing benign pigmented lesions from melanoma. Clin Exp Dermatol 15:167-71.

Lee KT, Gallagher R, Coldman A, McLean D (1997). Dullrazor: A software approach to hair removal from images. Comput Biol Med 27:533-43.

Lee KT (2001). Measuring border irregularity and shape of cutaneous melanocytic lesions. PhD, Simon Fraser University, Canada.

Loève M (1998) Fonctions aléatoires de second ordre. Levy P, editor, Processus Stochastiques et Mouvement Brownien, Hermann.

Lorentzen H, Wiesmann K, Kenet RO, Secher L, Larsen FG (2000). Comparaison of dermatoscopic ABCD rule and risk stratification in the diagnosis of malignant melanoma. Acta Derm-Venereol 80(2):122-6.

Mandelbrot BB (1982). The fractal geometry of nature. New York: W.H. Freeman and Co.

$\mathrm{Ng} \mathrm{V}$, Lee $\mathrm{T}$ (1996). Measuring border irregularities of skin lesions using fractal dimensions. In SPIE Photonics China, Electronic Imaging and Multimedia Systems, Beijing, 64-72.

Pehamberger H, Steiner A, Wolff K (1987). In vivo epiluminescence microscopy of pigmented skin lesions. Pattern analysis of pigmented skin lesions. J Am Acad Dermtol 17(4):571-83.

Pentland A (1984). Fractals: A model for both texture and shading. In Hoffman (Eds) Annual Meeting of the Optical Society of America: MIT. 
Rangayyan RM, El-Faramawy NM, Desautels J, Alim OA (1997). Measures of acutance and shape for classification of breast tumors. IEEE Trans Med Imaging, 16(6):199-210.

Ripley BB (1996). Pattern recognition and neural networks. Cambridge University Press.

Rumelhart DE, Hinton GE, Williams RJ (1988). Learning internal representations by error propagation. In parallel distributed processing - explorations in the microstructure of cognition, 1:318-62.

Sboner A, Blanzieri E, Eccher C, Bauer P, Cristofolini M, Zumiani G, Forti S (2001). A knowledge based system for early melanoma diagnosis support. Proceedings of the $6^{\text {th }}$ IDAMAP workshop - Intelligent Data Analysis in Medicine and Pharmacology, London, UK.

Schmid-Saugeon P, Guillod J, Thiran JP (2003). Towards a computer-aided diagnosis system for pigmented skin lesions. Computerized Medical Imaging and Graphics., 27:65-78.

Sonka M, Hlavac V, Boyle R (1993). Image processing, analysis and machine vision. London: Chapman \& Hall.

Stolz W, Braun-Falco O, Bilek P, Landthaler M, Cognetta AB (1994). Color atlas of dermatoscopy. Berlin: Blackwell Science Ltd.
Tamura H, Mori S, Yamawaki T (1978). Textual features corresponding to visual perception. IEEE Trans Syst Man and Cyb 8:460-73.

Taouil K, Bouhlel MS, Elloumi, Kammoun L (2002). Quantification des caractèristiques de mélanomes en vue d'une classification. JTEA'2002, Sousse Nord, Tunisia, 179-85.

Voight H, ClaBen R (1995). Topodermatographic image analysis for melanoma screening and the quantative assessment of tumor dimension parameters of the skin. Cancer 75(4):981-8.

Zagrouba E, Barhoumi W (2002). Semiautomatic detection of tumoral zone. Image Anal Stereol 21(1):13-8.

Zagrouba E, Barhoumi W (2003a). Objective and costefficient approach for skin lesions classification. Proceedings of the IEEE/ACS International Conference on Computer Systems and Applications, Gammarth, Tunisia, 135-6.

Zagrouba E, Barhoumi W (2003b). Systme d'aide au diagnostic du mélanome pour les dermatologues. $1^{\text {ere }}$ Conférence Francophone en Gestion et Ingénierie des Systèmes Hospitaliers, GISEH'2003, Lyon, France, 616-25. 